\title{
LOS SISTEMAS TRANSVERSALES Y SU IMPACTO EN LA EFICIENCIA Y EFICACIA DEL PODER JUDICIAL
}

\author{
JuAn CARlos MONTOYA MuÑOZ*
}

\begin{abstract}
Resumen
Los Sistemas Transversales se constituyen en las líneas maestras que informan las actividades de rutina de una organización, sea pública o privada. El presente artículo plantea la forma en que los Sistemas Transversales, tales como: administración financiera, planificación pública, participación ciudadana, supervisión y monitoreo, y reforma del Estado, informan el desenvolvimiento del Poder Judicial y el impacto que generan en dicho Poder del Estado. El fortalecimiento de los Sistemas Transversales y su aplicación práctica en el Poder Judicial, pueden dotarlo de eficiencia (capacidad para lograr un fin, empleando los mejores medios posibles) y eficacia (capacidad de lograr el efecto que se desea o se espera, sin que primen para ello los recursos o los medios empleados), en la medida que se articule con su visión, misión, plan estratégico, clima laboral y relación cohesionada con las demás organizaciones que componen el sistema de justicia.
\end{abstract}

Palabras clave: Sistemas Transversales - Sistema Transversal y Poder Judicial.

\begin{abstract}
Transversal Systems are master lines that report the routine activities of a public or private organization. This article addresses the form and manner in which the Transversal Systems, such as Financial Management, Public Planning, Citizen Participation, Supervision and Monitoring and State Reform provide insight on the performance of the Judiciary and on their impact in said State Branch. The strengthening of the Transversal Systems and their practical application in the Judiciary can endow it with efficiency (the capacity to achieve a goal making use of the best possible means) and efficacy (the capacity to achieve the desired or expected effect regardless of the resources or means used) as long as they are articulated with its vision, mission, strategic plan, working environment and its cohesive relationship with the remaining organizations of the justice system.
\end{abstract}

Key words: Transversal Systems - Transversal System and the Judiciary.

\section{Sumario}

1. Introducción. 2. Definición de sistemas transversales. 3. Tipos de sistemas transversales y su relación con las actividades del Poder Judicial. 3.1. Administración financiera. 3.2. Planificación pública. 3.3 Participación ciudadana. 3.4. Supervisión y monitoreo. 3.5. Reforma del Estado. 4. Conclusiones.

\footnotetext{
* Juez Titular Especializado en lo Contencioso Administrativo de la Corte Superior de Justicia de Lima - Poder Judicial del Perú.
} 


\section{INTRODUCCIÓN}

La Administración Pública, permite acercarnos los conceptos de políticas públicas y de gestión pública, entendido el primero como la definición de los fines o propósito del Estado con respecto a un conjunto de expectativas ciudadanas, y, el segundo, como manejar las ideas o buenos deseos en la acción real del Estado.

Las formas en que la Administración Pública se va relacionando en su rol como gestor de lo público, nos ubica en el marco del valor público, la gestión estratégica y la nueva gestión pública. Precisamente, es a través de este último concepto que se busca dar una nueva orientación a las organizaciones públicas, conocida también con el término de "nueva gerencia pública" o "New Public Managament - NPM", el cual describe una tendencia global hacia cierto tipo de reforma administrativa, dando una visión económica y gerencial de la administración gubernamental e involucrando otras necesidades, dependiendo del contexto.

En ese acercamiento entre las políticas públicas y la gestión, reconocemos la multidimensionalidad y complejidad de las mismas, que parten de la aplicación de sus procesos, entre ellos los sistemas transversales, tales como: Administración financiera, Planificación pública, Participación ciudadana, Supervisión y monitoreo y Reforma del Estado.

Al asociar dichos sistemas con el Poder Judicial, apreciamos el panorama en que se desenvuelve dicha organización; sin embargo, la mejora y fortalecimiento de cada uno de ellos, nos pueden conducir a la eficiencia y eficacia de dicho poder del Estado, tomando en cuenta la complejidad de su estructura, pues integra, en primer lugar, a los tribunales que imparten justicia, la que opera con independencia, como una precondición básica de funcionamiento y en segundo lugar, contiene una serie de órganos administrativos que apoyan el trabajo jurisdiccional y permiten que la misión de la institución se pueda cumplir.

El presente trabajo da una definición de sistemas transversales, estudia los tipos de sistemas transversales y su relación con las actividades del Poder Judicial, analizándose así, la Administración financiera., Planificación pública, Participación ciudadana, Supervisión y monitoreo y Reforma del Estado, y finalmente se esbozan las respectivas conclusiones. 


\section{DEFINICIÓN DE SISTEMAS TRANSVERSALES}

Los sistemas transversales se constituyen en las líneas, ejes o áreas que funcionan como información de las actividades de rutina de una organización.

\section{TIPOS DE SISTEMAS TRANSVERSALES Y SU RELACIÓN CON LAS ACTIVIDADES DEL PODER JUDICIAL}

\subsection{Administración financiera}

Tomando como referencia la definición de Marcos MAKÓN ${ }^{1}$ y la de José MENDOZA $^{2}$, la administración financiera significa para el Poder Judicial: Una secuencia de estrategias y acciones interrelacionadas orientadas a gestionar los fondos asignados y aplicarlos para alcanzar los objetivos y metas planificadas y presupuestadas por el Poder Judicial, en concordancia con sus funciones constitucionales.

La administración financiera del Poder Judicial articula un conjunto de componentes como son principios, normas, organismos, recursos, sistemas y procedimientos que intervienen en las operaciones de programación, gestión y control relacionadas con los ingresos asignados y gastos programados. Estos componentes en el caso del Poder Judicial, son los siguientes: (i) principios, (ii) normas, (iii) unidades orgánicas, (iv) recursos, (v) sistemas administrativos y (vi) procedimientos administrativos.

La Administración financiera en el Poder Judicial funciona como un sistema integrado a un sistema nacional denominado SIAF que comprende órganos rectores como el Ministerio de Economía y Finanzas, del cual el Poder Judicial es pliego conformado por diversas unidades ejecutoras.

El impacto que tiene una adecuada administración financiera en el Poder judicial redunda en la eficiencia y transparencia del servicio que presta, así como en la gestión y control de los ingresos y gastos; permitiéndonos responder a preguntas, como: ¿Cuál es el presupuesto?, ¿Cuál es el calendario de compromiso mensual de gastos del que se dispone?, ¿Quién gasta?, ¿En qué se gasta?, ¿Para qué se gasta?, ¿Cuánto se gasta?, ¿Qué actividad o proyecto corresponde el gasto?

1 MAKON, Marcos. «Sistemas integrados de administración financiera pública en América Latina». Santiago de Chile: ILPES- CEPAL, Marzo 2000, p. 11.

2 Mendoza Hernández, José. «Gestión Financiera Pública». Lima / Bogotá: Universidad Externado de Colombia, 2007, p. 25. 
En el Poder Judicial quien asume la responsabilidad de la administración financiera es la Gerencia General, al actuar como un Órgano Técnico y de apoyo del Consejo Ejecutivo del Poder Judicial.

Cabe señalar que una de las Unidades ejecutoras del Poder Judicial, la detenta el Proyecto de Mejoramiento de los Servicios de Justicia (PMSJ), la misma que gracias a un convenio celebrado por el Estado Peruano con el Banco Mundial, ha canalizado una donación de 12 millones de euros que desde febrero del 2005 viene empleándose en la capacitación de los jueces a nivel nacional, entre otros proyectos, lo que de alguna manera explica en qué se viene gastando dicho dinero.

\subsection{Planificación pública}

Para una adecuada y acertada planificación de una institución u organización pública, se tiene que realizar un estudio estructural de su funcionamiento y la repercusión que ésta tiene en la sociedad.

Una de las metodologías más útiles para el proceso de planificación y evaluación de programas es el denominado modelo lógico, que consiste en una forma sistemática y visual de presentar y compartir la comprensión de las relaciones entre los recursos que dispone para operar su programa, las actividades que planea realizar y los cambios o resultados que espera obtener ${ }^{3}$.

Hablar de planificación en el Poder Judicial implica pues la realización de un plan estructurado que supone la consecución de objetivos y metas desde un plano interno y externo, dependiendo del plazo que se establezca: corto, mediano o largo plazo.

Luis Lira plantea que la revalorización de la planificación alcanza a todas las instituciones y que cada una de ellas la utiliza de acuerdo a sus fines, enfatizando algunas concepciones teóricas y metodológicas y utilizando algunos instrumentos y descartando otros, lo que se traduce en aplicaciones muy diferentes. Señala además que no obstante quien sea el usuario, para nuestro caso el Poder Judicial, debe enfatizarse la concepción estratégica, tanto del diseño como de la ejecución, el obligado carácter participativo de ambos subprocesos y la necesidad de asegurar la anticipación del futuro, la coordinación de las acciones y la evaluación de los resultados ${ }^{4}$.

3 W.K. KelLOGG FoundATION. «Uso de modelos lógicos para integrar la planificación, evaluación y acción». En: Guía de Desarrollo de Modelos Lógicos, Diciembre del 2001, p. 1.

4 LIRA, Luis. «Revalorización de la planificación del desarrollo». Chile: Instituto Latinoamericano y del Caribe de Planificación Económica y Social (ILPES), Agosto 2006 pp. 5 - 6. 
Muchas prioridades saltan a la vista en el Poder Judicial, entre ellas la disminución de la sobrecarga procesal, de la morosidad, la implementación de una política anticorrupción, el avance tecnológico, etc. Sin embargo, para hacer realidad una adecuada planificación, necesitamos contar con un grupo de especialistas en gestión pública que se integren como directivos de la Institución a fin que contando con capacidad, competencia y liderazgo, promuevan el cambio.

$\mathrm{Al}$ respecto, considero un gran acierto la creación de los gerentes públicos ${ }^{5}$, los mismos que luego de una selección y capacitación, vienen incorporándose en los distintos niveles de la administración pública; tal como ha acontecido con el grupo de profesionales, que actualmente viene desempeñándose como administradores, de algunas Cortes Superiores de Justicia del País.

Los elementos a tomar en cuenta para una adecuada planificación en el Poder Judicial serían básicamente los recursos: económicos, humanos y logísticos.

Desde nuestra experiencia hemos podido apreciar que a fines del 2008, se realizó por primera vez en la historia del Poder Judicial, talleres descentralizados de validación del Plan Estratégico Institucional en todos los Distritos Judiciales, con la participación de todos los magistrados, proceso que culminó con tres talleres macro regionales en Ayacucho, Piura y Lima y un taller con los máximos directivos de la institución (miembros del CEPJ y jueces supremos). Ello constituye un gran paso en la tarea de planificar en el Poder Judicial.

\subsection{Participación Ciudadana}

La participación ciudadana en las instituciones públicas es una manera de fiscalizar las actividades que estas realizan, habida cuenta que los servicios y bienes son de naturaleza pública.

En el Poder Judicial dicha participación debería estructurarse sobre la base de la (i) información, (ii) la consulta y (iii) la participación activa de la ciudadanía, las mismas que a decir de Marc Gramberger siempre han existido, pero que sin embargo dado que la democracia no cesa de evolucionar, las reivindicaciones de apertura y transparencia de los ciudadanos, así como la naturaleza cada vez más compleja de las sociedades y de los retos que éstas

5 Decreto Legislativo No 1024 "Crea y regula el Cuerpo de Gerentes Públicos", publicado en el Diario Oficial "El Peruano" el 21/06/2008. 
enfrentan, llevan a los gobiernos a buscar, cada vez más, el fortalecimiento de estas interacciones ${ }^{6}$.

En el Poder Judicial el ciudadano puede tener acceso a la información institucional (documentos de gestión institucional) información sobre el personal y magistrados del Poder Judicial y sobre las resoluciones que emiten los órganos jurisdiccionales del país, así como hacer el seguimiento del estado de los expedientes judiciales vía el Sistema Integrado Judicial, hecho que incluso ha merecido un reconocimiento, al otorgársele el premio en la categoría Transparencia y acceso a la información en el concurso Buenas Prácticas en Gestión Pública 2011, organizado por Ciudadanos al día7.

El Poder Judicial ganó este premio por su propuesta "Consulta de expedientes judiciales en Lima", servicio que pretende resolver el problema del desconocimiento del estado del proceso judicial y las prácticas que dicha desinformación origina.

Asimismo el Poder Judicial facilita toda aquella información relacionada a los servicios judiciales (registro de deudores alimentarios morosos - Redam, registro de condenas, etc.) así como la solicitada por escrito (correo electrónico) con la salvedad de las excepciones establecidas en la Constitución y las leyes; información que debe ser proporcionada de manera oportuna y actualizada.

En el ámbito de la consulta ciudadana aparecen el derecho de petición, a través del cual los ciudadanos pueden hacer consultas al Poder Judicial o formular objeciones (recursos de impugnación) vinculados a asuntos que les afecten directamente, de conformidad con la Ley 27444 que aprueba la Ley General de Procedimiento Administrativo, que en el presente caso se encuentra regulado específicamente en el TUPA del Poder Judicial.

En el ámbito de la participación ciudadana en el Poder Judicial, cabe mencionar que, en armonía con la Ley de los Derechos de Participación y control ciudadanos, se promulgó en el año 2004 la Ley $N^{\circ}$ 28149, Ley que incluye la participación ciudadana en los órganos de control del Poder Judicial y del Ministerio Público, por lo que la Oficina de Control de la Magistratura

\footnotetext{
6 Gramberger, Marc. «Participación ciudadana». Manual de la OCDE sobre información, consulta y participación en la elaboración de políticas públicas OCDE (Organización para la cooperación y el desarrollo económicos). En: Lecturas Módulo 3 T. 1 para la Maestría en Derecho con mención en Política Jurisdiccional, Gestión y Política judicial. Lima: PUCP, p. 18.

7 Ciudadanos al Día, dio a conocer los ganadores de la séptima edición del Premio a las Buenas Prácticas de Gestión Pública 2011. Disponible en Internet: www.ciudadanosaldia.org/premiobpg2011/
} 
- OCMA, en cumplimiento de dicha norma, elaboró un nuevo Reglamento de Organización y Funciones a fin de adecuarla a la disposición normativa.

Siendo la corrupción uno de los problemas más graves y recurrentes que ensombrecen la loable labor del servicio de justicia, el ámbito judicial es una de las áreas más productivas para que la población, mediante el mecanismo de la información, conozca los alcances de la política de lucha contra la corrupción dentro del Poder Judicial. Una vez informada, la ciudadanía puede ser un elemento importante que puede coadyuvar a luchar contra este gran problema que se encuentra presente no solo en el Poder Judicial, sino también en los demás estamentos del Estado, así como en el ámbito privado.

\subsection{Supervisión y monitoreo}

El sistema de Supervisión y Monitoreo es un conjunto de normas, procedimientos, instrumentos y aplicaciones que se combinan a fin de valorar las acciones de una Organización en base a decisiones asumidas, cumplimiento de acuerdos, logros y resultados, con el propósito de mejorar y corregir su eficiencia.

A decir de Keith MACKAY existen varios términos y conceptos relacionados dentro del campo del seguimiento y evaluación a los gobiernos y que pueden prestarse a confusiones; señala además que cada país o agencia de desarrollo utiliza una definición diferente para el mismo concepto, lo que no ocurre con los mecanismos de medición que resultan comunes, así el caso de:

(i) Los indicadores de desempeño, (ii) evaluación previa, (iii) evaluación rápida, (iv) evaluación de impactos y (v) las revisiones exhaustivas de gastos.

Agrega el autor que dicho sistema puede ofrecer información exclusiva acerca del desempeño de las políticas, programas y proyectos de un gobierno y que su valor no es intrínseco sino que radica en "el uso de la información para ayudar a mejorar el desempeño del gobierno"8.

En lo que atañe al Poder Judicial y de acuerdo con el Plan de Gobierno del Poder Judicial $2011-2012^{9}$, dicho sistema podría estructurarse de la siguiente manera:

\footnotetext{
8 MACKAY, Keith. «Cómo crear sistemas de S y E que contribuyan a un buen gobierno», Independent Evaluación Group, Banco Mundial. En: Lecturas Módulo 3 T. 1 para la Maestría en Derecho con mención en Política Jurisdiccional, Gestión y Política judicial. Lima: PUCP, p. 7-8.

9 Plan de Gobierno del Poder Judicial 2011 - 2012; Disponible en internet: www.justiciaviva.org.pe/userfiles/ plan.pdf
} 


\section{A. Sistema de monitoreo}

(i) Objetivos que persigue: dar información sobre el cumplimiento de la planificación operativa, para hacer correcciones sobre.

(ii) Cronograma de actividades.

(iii) Fuentes: tomar en cuenta los instrumentos; indicadores (valoración cuantitativa del desempeño, cumplimiento, avance y progreso; procesos y productos) y evaluaciones (estudios, exámenes, verificación, informes de equipo técnico).

(iv) Frecuencia: Situación actual y fecha estimada de monitoreo, $\operatorname{Vgr}^{\circ}$ mensual, trimestral, semestral.

(v) Equipo profesional técnico y estructura de mando y responsabilidades.

(vi) Presupuesto asignado.

\section{B. Sistema de evaluación}

(i) Objetivos que persigue; determinar los logros en términos de efecto e impacto y compararlos con lo propuesto en los resultados.

(ii) Medición de cumplimiento de objetivos en términos de pertinencia, eficacia, eficiencia, impacto y sostenibilidad.

(iii) Fuentes: informes de monitoreo y seguimiento de los indicadores de efecto e impacto, información externa.

(iv) Frecuencia: Situación actual y fecha estimada de conclusión; Anual, final.

(v) Equipo de proyecto y/o especialistas externos; $\mathrm{Vgr}^{\circ}$ la sociedad.

(vi) Presupuesto asignado ${ }^{10}$.

Las características que debería tener ese sistema, son:

1. Pertinencia.- Capacidad de proporcionar información que se precisa o demanda para adoptar decisiones fundamentadas.

2. Veracidad.- Asegurar que la información corresponda a la realidad del proyecto, programa o institución que se observa.

3. Oportunidad.- Lograr que la información esté disponible en el momento en que lo requiere el proceso de toma de decisiones.

4. Legitimidad.- Que la información y los juicios que el sistema genere sean respetados por los actores relevantes, sustentando las decisiones que de ellos se desprendan.

\footnotetext{
${ }^{10}$ INFORMET. «Taller para el diseño de metas e indicadores del plan estratégico del Poder Judicial», Metodología del sistema de medición de la planificación estratégica; Noviembre del 2008; Disponible en internet: $\underline{w w w}$. pj.gob.pe.
} 
5. Eficiencia.- Que la información se genere al menor costo posible.

6. Transparencia.- en la obtención y emisión de datos.

7. Precisión.- En los objetivos y fines a supervisar.

Este sistema debería evaluar y monitorear:

1. Sobre la utilización del presupuesto del Poder Judicial.

2. Sobre el desempeño del personal.

3. Sobre los fallos contradictorios.

4. La corrupción en el Poder Judicial.

5. Implementación de infraestructura y equipamiento.

6. La morosidad procesal.

7. Sobre la producción cualitativa y cuantitativa de las resoluciones judiciales.

\subsection{Reforma del Estado}

La Reforma de la administración pública centra su atención en la gestión pública y en la forma como elevar la capacidad institucional del Estado para diseñar, implementar y evaluar políticas que efectivamente generen valor público en un contexto democrático ${ }^{11}$.

Una iniciativa de reforma dentro del Poder Judicial debe contar con características como: Debe ser plural y democrática, transparente, participativa, técnica, objetiva e independiente, centrada en el usuario del servicio judicial, contar con agentes participativos y eficientes, contar con un presupuesto acorde a las necesidades de la reforma propuesta, así como un sistema de supervisión y control de dicho presupuesto asignado.

Las principales razones para que los esfuerzos de reforma no hayan tenido éxito hasta el momento se deben:

a. A la ausencia de voluntad política: por ejemplo en la modificación y dación de normas legales;

b. La falta de articulación en cuanto a sus objetivos estratégicos de las Entidades comprendidas en el Plan Nacional para la Reforma de la Administración de Justicia de CERIAJUS;

c. La ausencia de recursos; los cuales se aprecian en el área de infraestructura, en el aspecto remunerativo de los ingresos de los trabajadores del Poder

11 Cortazar Velarde, Juan Carlos. «La reforma de la Administración Pública Peruana, 1990-1997». En: Lecturas Módulo 3 T. 1 para la Maestría en Derecho con mención en Política Jurisdiccional, Gestión y Política Judicial. Lima: PUCP, p. 1. 
Judicial, y la mejora consiguiente de las políticas de recursos humanos al interior de la institución, que vele por un buen clima laboral y también por una cultura ética;

d. La falta de una reforma educativa en materia jurídica;

e. La falta de control disciplinario de los Colegios de Abogados a sus agremiados;

f. Un mayor compromiso de la propia judicatura con la reforma judicial.

La reforma judicial debe ser dirigida o liderada por el Poder Judicial, con la cooperación de otros actores como el Congreso de la República, el Poder Ejecutivo y la Sociedad Civil.

\section{CONCLUSIONES}

1. El fortalecimiento de los Sistemas Transversales y su aplicación práctica en el Poder Judicial, pueden dotarlo de eficiencia y eficacia, en la medida que se articule con su misión (quiénes somos), visión (cómo queremos que nos vean), plan estratégico (estrategias, objetivos, tácticas y acciones), clima laboral (compromiso del personal) y mantener una relación cohesionada con las demás Organizaciones que componen el sistema de justicia (multidimensionalidad).

2. La correcta aplicación de los sistemas transversales referidos, genera grandes beneficios en el ciudadano, básicamente por el hecho que cada uno de ellos alimenta o sirve como fuente de información para que el Poder Judicial responda con eficiencia y eficacia en el servicio que presta: La Justicia. 